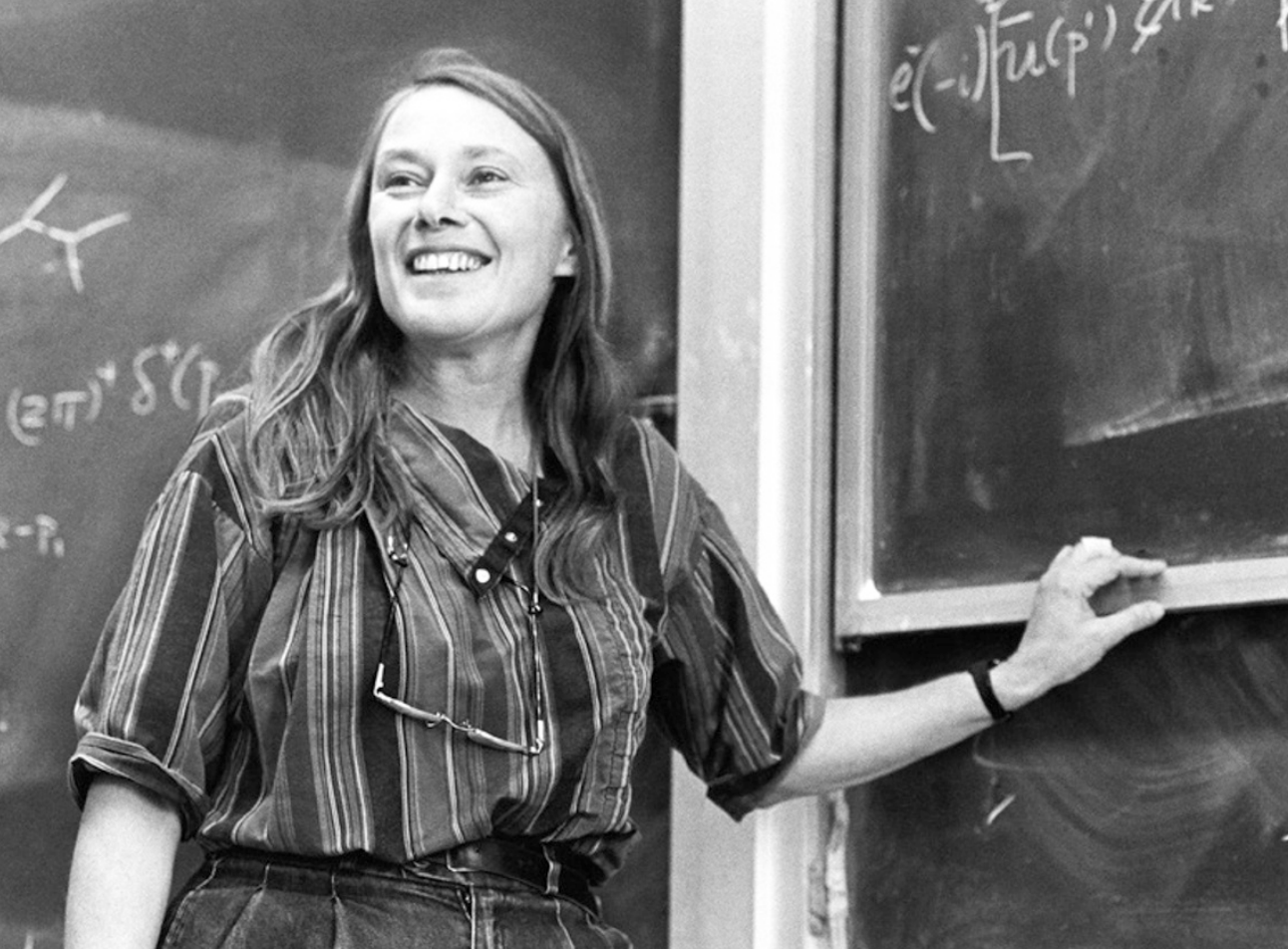

Mary K. Gaillard's theoretical-physics achievements include predicting the mass of the charm quark.

PHYSICS

\title{
She did it all
}

\section{Val Gibson enjoys the autobiography of Mary K. Gaillard, the first female physics professor at Berkeley.}

\section{$\mathrm{T}$} he brilliant theoretical physicist Mary K. Gaillard has made major contributions to the standard model of particle physics and to superstrings, a candidate theory of everything. In 1981, she became the first woman with a tenured position in the physics faculty at the University of California, Berkeley. Her frank autobiography, A Singularly Unfeminine Profession, is an honest, revelatory account of her many discoveries, made as she battled gender bias and faced the demands of raising three children.

Born in New Jersey in 1939, Gaillard has a "survival mechanism" born from an inherent belief in equality, nurtured by her parents and school, and a rebellious tendency to question the world around her. Having fallen in love with physics at school, she won a scholarship to Hollins College near Roanoke, Virginia. It included a year in Paris at l'École Polytechnique - her first exposure to the culture that was to become her nemesis.

During college, Gaillard also spent two summers at Brookhaven National Laboratory in Upton, New York, where she became hooked on high-energy particle physics. There, she met her first husband: Jean-Marc Gaillard, a postdoc at Columbia University in New York City. She did a graduate year at Columbia, then Jean-Marc was offered a post in Orsay near Paris. His colleagues advised Gaillard to accompany him, and to become "self-taught, like all great European

physicists". The first year in Orsay became "the worst year", as Gaillard "learnt to be a housewife" and was largely left on her own.

Jean-Marc was then offered a six-year staff position at CERN, the European centre for particle physics near Geneva, Switzerland. Here, Gaillard became a long-term visitor for some 20 years. Through Jean-Marc's connections, she secured space in a shared basement office in the CERN theory group; commuted between Orsay and CERN; and worked on the difference between matter and antimatter.

She was subjected to the "determined antifeminism" of theory-group leader Leon Van Hove, who became CERN directorgeneral. He would ignore her and ask male colleagues about a project, for instance. Juggling research and her children, Gaillard became a major player in theoretical particle physics. Without the flexibility to interact with colleagues, she wrote many early papers alone. It is easy to sympathize when she tells of forgetting to collect her eight-year-old son Bruno from a music lesson in midwinter, or giving him the wrong bus fare so he was fined.

In 1973, Gaillard spent a "pivotal year" as

A Singularly Unfeminine

Profession: One Woman's Journey in Physics MARY K. GAILLARD World Scientific: 2015 a visitor at Fermilab near Chicago, Illinois, which was buzzing with excitement about the proposed theory of weak interactions. She met Benjamin Lee, with whom she predicted the mass of the charm quark, gaining a "sort of star status". Back at CERN and working with giants such as John Ellis and Dimitri Nanopolous, she turned to the decay modes of the Higgs boson, the signature for gluons and the mass of the bottom quark. She co-authored the paper that introduced the term penguin diagram for a type of loop-containing Feynman diagram (a description of interactions between subatomic particles). The same paper earned Bruno, then nine, an acknowledgement for "help with the calculations".

When Gaillard headed back to the United States in 1981 for the tenured position at Berkeley, it was not with Jean-Marc but with her soon-to-be second husband Bruno Zumino, a supersymmetry theorist who died last year. Gaillard became a grande dame of particle physics, with positions on many committees that shaped particle-physics research in the United States and, ultimately, the world.

The story is as much about a thrilling period in particle physics as about Gaillard's struggle to establish herself in a male-dominated sphere. Hers is the era of the standard model and its description of fundamental particles and forces. It has also seen the discovery of the Higgs boson, "a bug sloshing through molasses" as Gaillard describes it.

Gaillard explains her contributions clearly and without equations; exquisite illustrations by her son Bruno are reproduced. A fine example is the paper, presented as a conversation between herself, Lee and Jonathan Rosner, that used indirect experimental observations to predict the mass of the charm quark - three months before it was discovered (M. K. Gaillard et al. Rev. Modern Phys. 47, 277-310; 1975). Now 76, Gaillard continues to add to her broad portfolio, with a focus on superstrings and a desire to link theoretical predictions to experimental observation.

In 1980, Gaillard produced the Report on Women in Scientific Careers at CERN. This addressed the fact that just 3\% of CERN staff were women, and called for the elimination of gender discrimination through equality in promotion, maternity leave and provision of a full-day crèche. Only in 1994 was a female experimental physicist, Fabiola Gianotti who will become CERN director-general in 2016 - appointed to a senior position. Unfortunately, the current list of physicists in the CERN theory group shows no women in permanent senior positions. This is no reflection on Gaillard. As a colleague comments in the book: "She did it all!" -

Val Gibson is an experimental particle physicist who has worked at CERN near Geneva, Switzerland. She is head of highenergy physics in the Cavendish Laboratory at the University of Cambridge, UK, and is a champion of equality and diversity. e-mail:gibson@hep.phy.cam.ac.uk 